\title{
A simplified hydrokinetic model for a steady-state microwave discharge sustained by traveling waves at atmospheric pressure conditions
}

\author{
F. J. Gordillo-Vázquez and J. Cotrino ${ }^{a)}$ \\ Departamento de Física Atómica, Molecular y Nuclear, Facultad de Física, Universidad de Sevilla, \\ Apartado 1065, 41080 Sevilla, Spain
}

(Received 17 November 1994; accepted for publication 16 June 1995)

\begin{abstract}
The properties of a microwave-induced argon plasma produced by traveling surface wave at atmospheric pressure are investigated theoretically. A hydrokinetic model is elaborated to obtain the nonequilibrium one-dimensional profiles of electron temperature $T_{e}$ (average electron energy), electron density $n_{e}$, and the first excited state population density $n_{4 s}$, along the axis of a steady-state discharge. A three-level atomic structure is assumed for the argon atom. A particle balance is included through the continuity equations for $n_{e}$ and $n_{4 s}$. These equations are coupled with an energy balance equation for the electrons. The effects of different parameters on the properties of the argon discharge are investigated: discharge tube radius, gas flow rate, resonant radiation-escape factor, and neutral gas temperature. (c) 1995 American Institute of Physics.
\end{abstract}

\section{INTRODUCTION}

Microwave-induced argon plasmas (MIP) have become extensively used as particularly attractive atomic emission sources because of their high efficiency; they can excite atomic and ionic species. ${ }^{1,2}$ An interesting feature of this type of discharge is that the process of energy transfer from the electromagnetic field of the wave to the plasma takes place along the same direction as that of the traveling wave. ${ }^{3}$ In this article we assume that the plasma column is sustained in flowing argon at atmospheric pressure by the propagation of a surface wave, which is excited by a localized microwave gap-type structure called a surfatron. The principle of operation of this launcher has already been extensively described elsewhere. ${ }^{1,4}$ The surface-wave-produced plasma belongs to the class of if and microwave-induced plasmas. It results from the propagation of an electromagnetic wave which uses the plasma column it sustains and the plasma tube as its sole propagating media.

Many experimental and theoretical studies have been done on surface wave discharges at low and medium gas pressure conditions: studies of the possible attenuation mechanisms of the wave, experimental and theoretical study of the axial surface wave propagation, experimental measurements of the electron density, temperature, and different excited states, ${ }^{5,6}$ However, the axial behavior of a very widely used device (MIP maintained by surface wave at atmospheric pressure conditions) is not well understood from a theoretical point of view. Such devices are frequently used as efficient sources of ionization and excitation of atoms and molecules, mainly in plasma chemistry and elemental analysis. Over the last decade, experimental investigations and several applications of high-frequency discharges maintained by surface wave have been reported. ${ }^{7}$ The axial nonuniformity is the main intrinsic property of these plasma discharges. This creates a demand for appropriate models of the axial behavior of such discharges.

\footnotetext{
a)Electronic mail: microondas@cica.es
}

In these discharges the electron and gas temperature values are clearly different; this shows that these plasmas are nonequilibrium plasmas. Up to now, many noncollisionalradiative models proposed to describe if and microwave frequency discharges at atmospheric pressure have been devoted to inductively coupled plasmas (ICP). Moreover, very few authors have included in their models some processes that are responsible for the nonequilibrium state of the plasma. Concerning surface-wave-produced plasmas, a theoretical study of the axial surface wave propagation has been recently published. ${ }^{5}$ In that article the wavelength and the attenuation coefficients of a high-frequency ( $\mathrm{r}$ and microwave) surface wave of azimuthal symmetry are calculated as a function of the electron density along an atmospheric pressure plasma column. On the other hand, a theoretical model at atmospheric pressure condition, a channel-type model for If and microwave discharges, assuming local thermodynamic equilibrium and sustained by traveling waves, has also been proposed. ${ }^{8}$ This channel-type model includes the axially nonuniformity properties of such discharges.

The purpose of this article is to present a thcoretical study of the propagation of high-frequency (HF) surface wave of azimuthal symmetry, along an atmospheric pressure plasma column in nonequilibrium thermodynamic regime. In such a case, the collision frequency is typically such that $\nu>\omega$, where $\nu$ is the electron-neutral collision frequency for momentum transfer and $\omega$ is the wave angular frequency. We assume that the electron-neutral collisions are determining the attenuation of surface waves along the plasma column. We do not consider nonlinear effects connected with the heating of the electrons or with the ionization of neutral gas (no dependence of the plasma permittivity on the wave electric field intensity). We consider a simplified kinetic model for the argon plasma and an energy balance equation for the electrons. This model will allow us to find the nonequilibrium onedimensional profiles of the electron temperature $T_{e}$ (average electron energy), electron density $n_{e}$, and the first excited state population density $n_{4 s}$, along the axis of a steady-state argon discharge. The influence of different parameters (i.e., the gas flow rates, plasma radii, neutral gas 
1 Plasma

2 Glass

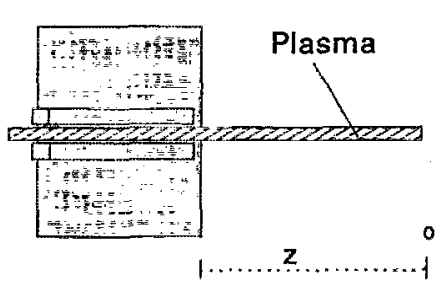

Axial position

a)

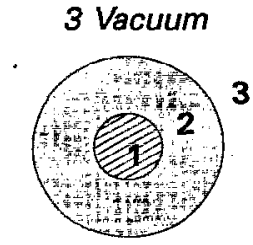

FIG. 1. Schematic of the (a) surface wave device and (b) propagation media considered for the calculation of the electric-field intensity.

temperature, and trapping of resonant radiation) on these calculated values is also investigated.

A schematic presentation of the propagating media considered in our calculations is shown in Fig. 1. A $2450 \mathrm{MHz}$ surfatron gap-type exciter is assumed which launches a surface wave that sustains an argon discharge at atmospheric pressure. The plasma forms near the gap and, depending upon input power supply and gas flow rate, extends outward several centimeters. Both the electron density and the HF power flux decrease gradually with increasing axial distance from the surfatron. The plasma column ends when the $\mathrm{HF}$ electric-field intensity drops below the threshold value necessary to sustain a steady-state discharge. We consider a plasma that completely fills the capillary quartz tube and which is totally located within the discharge tube. The behavior of the plasma sustained in a gas such as argon, for a tube of given inner diameter, is much influenced by the rate of gas flow used. For example, if the inner diameter of the tube is higher than $3-6 \mathrm{~mm}$ and the flow rate of argon is of several $\mathscr{C} / \mathrm{min}$, one observes experimentally a plasma composed of several filaments (two or three); however, with smaller flow rates (several hundred of $\mathrm{ml} / \mathrm{min}$ ), the plasma consists of a single filament, centered at the axis of the discharge tube, and touching the tube walls only near the gap. Here the plasma remains radially constricted to an apparent diameter of a little more than $1 \mathrm{~mm}$. Stable plasmas can also be generated in capillary tubes of a smaller diameter, but then either larger gas flow rates or thicker wall tubes should be used. In our theoretical model we assume that the plasma fills thoroughly the capillary tube.

The organization of this article is as follows. In Sec. II we describe the hydrokinetic model: HF electric-field intensity and model resolution. Section III shows the results obtained, and finally in Sec. IV the main conclusions are presented.

\section{HYDROKINETIC MODEL}

\section{A. HF electric-field intensity}

The aims of this subsection are mainly two: first the study of the wave propagation in the case where $\nu$ is not negligible with respect to $\omega$, and second the determination of the HF electric-field intensity as a function of the electron density profile and $\nu$. To treat the above problem in a selfconsistent manner requires simultaneously solving the Maxwell equations and the equations describing the discharge processes. In our calculations we consider one commonly used approximation, which is to solve separately the plasma maintenance equations and the equations describing the electrodynamic properties of the system (including the discharge), and only afterward to merge the results. Nowakowska and co-workers ${ }^{8}$ have developed a theoretical study of the propagation of a HF surface wave of azimuthal symmetry along an atmospheric pressure plasma column. They use the standard cold plasma surface wave formalism and their methods are used in this article. For example, the wave dispersion characteristics are computed using complex algebra, since, at atmospheric pressure, the plasma permittivity has comparable real and imaginary components. The cold plasma approximation seems appropriate since the electron temperature is estimated to be roughly $1 \mathrm{eV}$. On the other hand, we can assume that the collisions are mainly between electron and neutral atoms. Then, the plasma response, as far as wave propagation is concerned, can be readily described by the usual equivalent dielectric permittivity, which relatively to vacuum is expressed as

$$
\epsilon_{p}=1-\frac{\omega_{p e}^{2}}{\omega(\omega+j \nu)}=1-\frac{\left(\omega_{p e} / \omega\right)^{2}}{1+j(\nu / \omega)},
$$

where $j$ is the imaginary operator, $\omega$ is the wave frequency, and $\omega_{p e}$ is the plasma frequency $\left(=n_{e} e^{2} / m_{e} \epsilon_{0}\right)^{1 / 2}$. In our case, as it was mentioned before, we always have that the electron-neutral collision frequency for momentum transfer $\nu$ is larger than $\omega$. It must then be remembered that the conductivity that leads to Eq. (1) is derived from the model of an electron moving through a viscous medium, ${ }^{9}$ i.e., from the case where the electron makes many collisions during one oscillation period of the wave. This means that $\nu$ may be much larger than $\omega$ but it should, nevertheless, remain smaller than the plasma frequency $\omega_{p e}$ for the hydrodynamic model to be valid. The collision cross section is velocity dependent and $\nu$, in Eq. (1), has to be replaced by an effective collision frequency defined by

$$
\nu_{\mathrm{ef}}=\frac{\int_{0}^{\infty}\left[\nu \epsilon^{3 / 2} /\left(\nu^{2}+\omega^{2}\right)\right](d f / d \epsilon) d \epsilon}{\int_{0}^{\infty}\left[\epsilon^{3 / 2} /\left(\nu^{2}+\omega^{2}\right)\right](d f / d \epsilon) d \epsilon} .
$$

The effective collision frequency is obtained from Eq. (2) assuming a Maxwellian electron energy distribution function and $\nu_{\text {ef }}$ depends on the electron temperature. In Eq. (2) $\epsilon$ denotes electron energy. Figure 2 shows $\nu_{\text {ef }}$ as a function of electron temperature. Also, we consider that the plasma is macroscopically neutral and radially homogeneous since in capillary tubes the wave characteristics depend weakly on the radial electron density profile $n_{e}(r)$. The propagation media considered in our calculations.is shown in Fig. 1(b). It corresponds to a plasma that completely fills the capillary tube where the discharge takes place. The capillary tube permittivity and loss tangent are included in our calculations through a complex permittivity $\epsilon_{\mathrm{g}}$ :

Under these conditions, the Maxwell equations in each medium, with the appropriate boundary conditions (continu- 


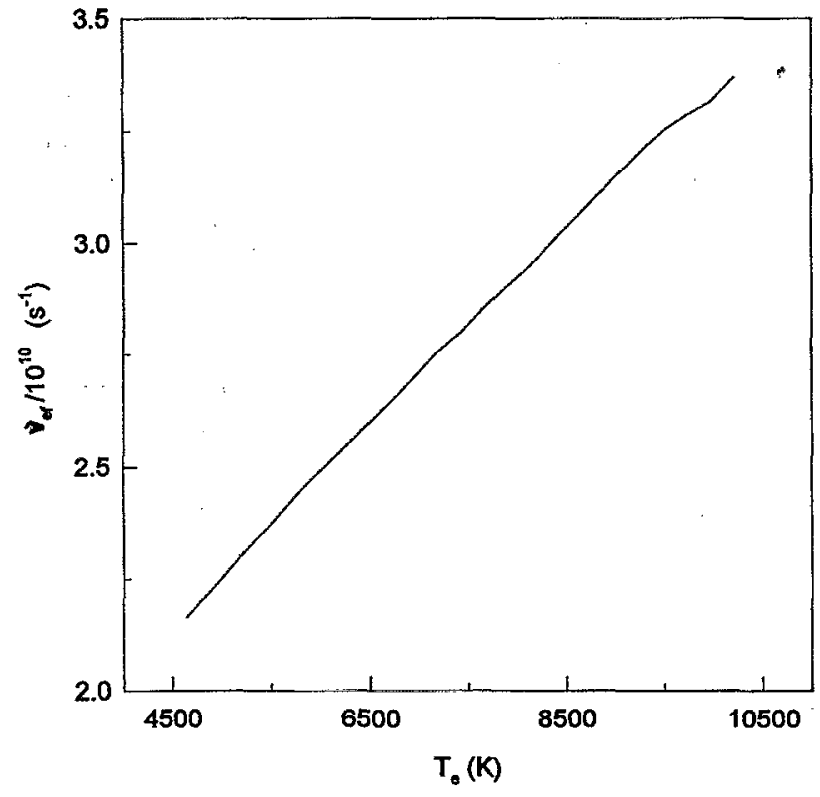

FIG. 2. Effective electron-neutral collision frequency for momentum transfer as a function of the electron temperature.

ity equation at the different interfaces), have been solved. Because the wave is azymuthally symmetric, the electromagnetic field has only three components: the axial, $E_{z}$ and radial $E_{r}$ electric fields and the azimuthal $H_{\varphi}$ magnetic field. The set of four continuity equations $\left[E_{z}\right.$ and $H_{\varphi}$ continuity at the plasma-quartz $(r=a)$ and at the quartz-air $(r=b)$ interfaces] has a single nonzero solution provided its determinant $(4 \times 4)$ vanishes, yielding the dispersion equation. ${ }^{10}$ The solution of the dispersion equation leads, for each value of $\nu_{\mathrm{e} f}(\omega$, to two curves: $\alpha$ (the attenuation coefficient) and $\beta$ (the wave number, $2 \pi / \lambda$ ) vs $\omega / \omega_{p e}$.

The electromagnetic-field components $\left(E_{r}, E_{z}, H_{\varphi}\right)$ can be obtained, in absolute form, if we use a normalization condition for the power flow $P$,

$$
P=\frac{1}{2} \operatorname{Re} \int_{S_{t}} E_{r} H_{\varphi}^{*} 2 \pi r d r
$$

where $S_{t}$ is the total system cross section $(0 \leqslant r \leqslant b)$ and $\operatorname{Re}$ means "real part." 11 We use in our model $E=\left[E_{z}^{2}(r=a)+E_{r}^{2}(r=a)\right]^{1 / 2}$ as the maintenance HF electric-ficld intensity. Figure 3 shows the behavior of the HF electric-field intensity $E$, as a function of the electron density $\left(\omega / \omega_{p e}\right)$ and electron temperature through the parameter $\nu_{\mathrm{ef}} d \omega$. In the electron density range of interest, we can see that the dependence of $E$ on the electron temperature $\left(\nu_{\mathrm{ef}}\right)$ is very weak. Then we consider hereafter, as a first approximation, that the HF electric-field intensity does not depend on $T_{e}\left(\nu_{\mathrm{ef}}\right)$, but only on the electron density. A more detailed analysis should allow for both dependences. The HF electricficld intensity for $\nu_{\mathrm{ef}} / \omega=1.76\left(T_{e}=0.6 \mathrm{eV}\right.$, this is an avcrage value of the experimental electron temperature reported in the bibliography) is the maintenance $\mathrm{HF}$ electric-field intensity considered in our calculations.

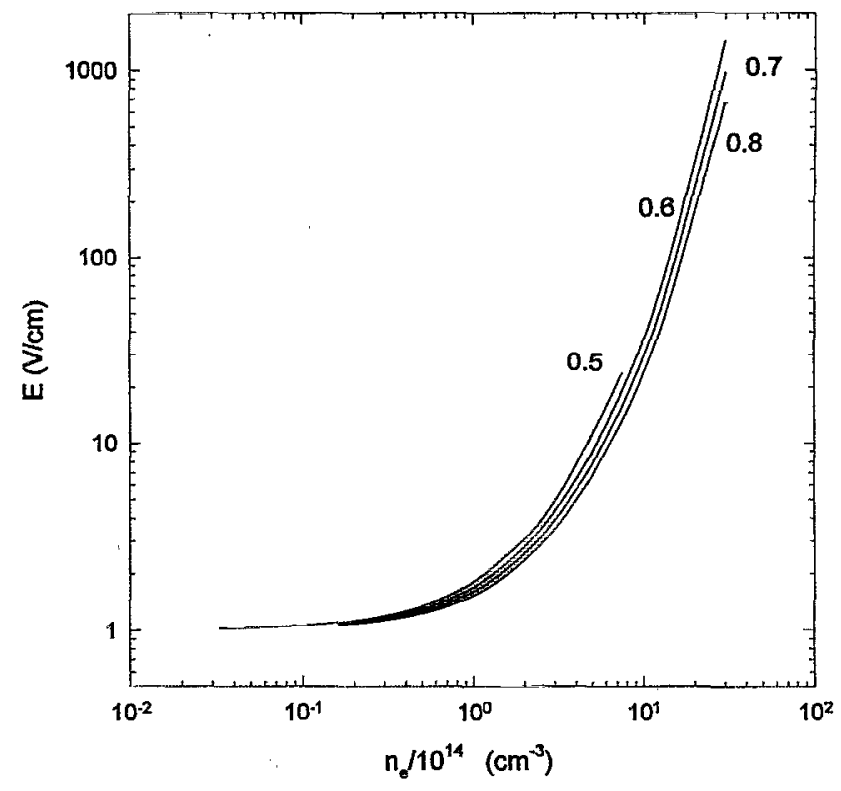

FIG. 3. Electric-field intensity as a function of the electron number density for different electron temperatures (in $\mathrm{eV}$ ).

\section{B. Three-level atomic model}

In the argon atomic structure, there is a significant energy gap from the four lowest excited electronic states $\left(3 p^{5} 4 s\right)$ to the upper excited states. ${ }^{12}$ Moreover, the energy separation between these four states is very small, so that a three-level atomic model ${ }^{13}$ (TLAM) can be used which includes the group of $4 s$ levels with the $3 s^{2} 3 p^{6}{ }^{1} s_{0}$ state as the ground level and the $3 s^{2} 3 p^{5}{ }^{2} P_{1 / 2}$ ionic state as the continuum. The three levels of the argon atom are denoted hereafter by $s$ (the ground state), $p$ (the excited state: two metastable levels, $3 p^{5} 4 s^{3} P_{0}$ and $3 p^{5} 4 s^{3} P_{2}$, and two resonant levels, $3 p^{5} 4 s^{1} P_{1}$ and $3 p^{5} 4 s^{3} P_{1}$ ) and $c$ (the continuum).

The choice of this simplified argon atom energy structure is related to the fact that the upper excited levels ( $4 p$ and above) remain much closer to their equilibrium valucs than the $4 s$ state, ${ }^{14}$ then the assumption of considering only the $4 s$ state is reasonably good, as a first approach, to the study of the nonequilibrium kinetics in an argon discharge.

We assume with a good approximation, since we have a low gas flow, that this flow is laminar; then for symmetry reasons the gas current lines are parallel to the axis $z$. On assuming laminar gas llow, the Navier-Stokes equations simplify. The main consequences for our model are that the flow velocity $u$ only depends on the radial coordinate $r$, and that the gas pressure $p_{g}$ only varies axially. Moreover, we suppose a linear decreasing behavior for the pressure along the discharge tube. The parabolic profile for the flow speed has the expression

$$
u(r)=\frac{2 \phi}{\pi R^{2}}\left(1-\frac{r^{2}}{R^{2}}\right),
$$

where $\phi$ is the gas flow rate and $R$ is the inner radius of the discharge cylinder. Since our calculations are carried out on the discharge axis, we take $u(r=0)$. 
We consider the equations

$$
\frac{\partial n_{i}}{\partial t}+\nabla \cdot\left(n_{i}\left(\mathbf{u}+\mathbf{U}_{i}\right)\right)=\dot{n}_{i}
$$

so that for $i(=s, p, e)$ we obtain the rate equations related to the ground level of argon, its first excited level, and the electron density, respectively. Here $\mathbf{U}_{i}$ and $\mathbf{u}$ represent the diffusion speed of species $i$ and the plasma speed when considered as a whole, respectively. In the same way, $\dot{n}_{i}$ accounts for the different collisional and radiative processes responsible of the changes in the population $n_{i}$. These three equations can be reduced to only two ${ }^{13}$ if we assume that the total number of particles $n_{a}$, is kept constant in each section of the plasma column,

$$
n_{a}=n_{e}+n_{p}+n_{s} .
$$

We choose to keep the equations related to the first excited level $(p)$ and the electrons $(c)$. Then we have

$$
\begin{aligned}
\dot{n}_{p}= & u \frac{\partial n_{p}}{\partial z}=n_{e} n_{s} k_{s p}+n_{e}^{3} k_{c p}+n_{e}^{2} A_{c p}^{\text {eff }} \\
& -n_{e} n_{p}\left(k_{p c}+k_{p s}\right)-n_{p} A_{p s}^{\text {eff }}, \\
n_{e}= & u \frac{\partial n_{e}}{\partial z}=n_{e} n_{s} k_{s c}+n_{e} n_{p} k_{p c}-n_{e}^{3}\left(k_{c s}+k_{c p}\right) \\
& -n_{e}^{2}\left(A_{c s}^{\text {eff }}+A_{c p}^{\mathrm{eff}}\right),
\end{aligned}
$$

which are the rate equations expressed in cylindrical coordinates, when there is only an axial dependence. Moreover, $U_{i}=0$ since we neglect the axial diffusion of any species present in the plasma. The quantities $k$ and $A^{\text {eff }}$, in Eqs. (7) and (8), represent the electron-impact collisional transitions and (effective) radiative transitions, respectively. Here $k_{s p}$ and $k_{p s}$ are rate integral coefficients for electron-impact excitation and deexcitation of the atomic level $p$, respectively; $k_{s c}\left(k_{p c}\right)$ and $k_{c s}\left(k_{c p}\right)$ are rate integral coefficients for electron-impact ionization of level $s(p)$, respectively. The quantities $A_{c l}^{\text {eff }}(l=s, p)$ in Eqs. (7) and (8) are the effective rate integral coefficients for free-bound transitions $(c \rightarrow p$ and $c \rightarrow s$ ). They consider, through the escape factors $\Lambda_{c l}$, the radiative recombination and photoionization processes, ${ }^{15}$ respectively; they measure the portion of radiation leaving the plasma. The escape factor is a number between zero (in an optically thick plasma for the spectral line produced in the transition) and one (in an optically thin plasma for the line). Plasma radiation that corresponds to spontaneous deexcitation $p \rightarrow s$ is hereafter denoted as resonance radiation. Since the density of absorbing ground-level atoms is relatively large, the escape factor $\Lambda_{p s}$, for resonance radiation, is often small. The quantity $A_{p s}^{\text {eff }}=\Lambda_{p s} A_{p s}$ is a measure of the radiative deexcitation of the excited level $p, A_{p s}$ being the Einstein coefficient for spontaneous emission. Its value $\left(3 \times 10^{8}\right.$ $\mathrm{s}^{-1}$ ) is taken as the average of the corresponding coefficients for the resonant transitions from ${ }^{1} P_{1}$ and ${ }^{3} P_{1}$ argon levels. The escape factors $\Lambda_{c p}$ and $\Lambda_{c s}$ are considered as parameters whose values are $\Lambda_{c p}=\Lambda_{c s}=1$ (thin plasma for level $c$ ), and $\Lambda_{p s}$ ranges from $10^{-6}$ to $10^{-3}$ (thick plasma for level $p$ ), since only resonant radiation trapping coming from the spontaneous deexcitation of level $p$ is considered. On the other hand, the reason to make the assumption that $\Lambda_{c p}=\Lambda_{c s}=1$ is rooted in the fact that the strong $c \rightarrow s$ and $c \rightarrow p$ radiative recombination not balanced by a corresponding photoionization will cause the plasma to be in nonequilibrium. Therefore, the electron density will be less than its thermodynamic equilibrium value, obtained through the Saha law using the electron temperatures given by the model, as is experimentally corroborated. ${ }^{5,6,16}$

\section{Electron energy balance equation}

The electron energy balance equation used in our calculations is obtained from the kinetic theory, as the third moment of the Boltzmann equation, and it reads ${ }^{17}$

$$
\boldsymbol{\nabla}\left(\frac{3}{2} n_{e} k_{B} T_{e} \mathbf{u}\right)+n_{e} k_{B} T_{e} \boldsymbol{\nabla} \cdot \mathbf{u}+\boldsymbol{\nabla} \cdot \mathbf{q}_{e}=Q^{e},
$$

where $n_{e}, \mathbf{u}$, and $T_{e}$ are the electron density, gas velocity, and electron temperature, $k_{B}$ is the Boltzmann constant, and $q_{e}$ is the heat flux related to the axial electron flow. The right-hand side of Eq. (9) represents the source term, i.e., all energy, excluding viscous dissipation and heat conduction, supplied to the electrons by collisions with other particles.

In our case, Eq. (9) is rather simplified since we are dealing with a steady-state plasma where both the inelastic collision energy losses (which we assume to be almost negligible at $p_{g}=1 \mathrm{~atm}$ ) and the viscous dissipation are not considered. However, the energy loss due to the radiative recombination processes $(c \rightarrow s, c \rightarrow p)$ is kept.

After simplifying, if we take only the axial component of Eq. (9) and knowing that $\partial u / \partial z=0$, we have

$$
n_{e} u \nabla_{z} \cdot\left(\frac{3}{2} k_{B} T_{e}\right)+\nabla_{z} \cdot \mathbf{q}_{e}=Q^{e} .
$$

In the following subsections, we describe the different source terms considered.

\section{Ohmic heating}

The electrons dissipate the ohmic power through collisions with the heavy particles; so the source term (the electron thermal energy increment per unit volume), due to the presence of an electric field, is given by

$$
Q_{0}^{e}=\mathbf{J}_{e} \cdot \mathbf{E}=\sigma E^{2},
$$

where $\sigma$ and $E$ represent the electron electrical conductivity in argon and the HF electric-field intensity, respectively.

\section{Elastic losses}

Because of the elastic collisions between heavy particles and electrons, the latter spend part of their energy and then we have that the source term related with the elastic power losses is

$$
Q_{\mathrm{el}}^{e}=-\frac{2 m_{e}}{M_{h}} \frac{3}{2} k_{B}\left(T_{e}-T_{g}\right) \nu_{\mathrm{eh}} n_{e},
$$

where $m_{e}$ and $M_{h}$ are the masses of electrons and heavy particles. In an argon plasma, the collision frequency for momentum transfer $\nu_{\text {en }}$ depends on the electron energy and since our study deals with HF discharges, we must use an effective collision frequency $\nu_{\mathrm{ef}}\left(T_{e}\right)$ given in Eq. (2). 


\section{Radiative recombination}

When an clectron meets an ion, both of them can recombine producing a neutral atom and a photon. During this process the total amount of thermal energy per unit volume does not change for the heavy particles, but it does change for the electrons since a thermal electron is eliminated. The recombination energy itself, $h \nu_{c s}$ or $h \nu_{c p}\left(\nu_{c s}\right.$ and $\nu_{c p}$ are the transition frequencies), which is also contained in the escaping photon, does not lower the thermal energy. Then the processes of spontaneous deexcitation of atoms do not need to be included in the electron energy balance equation. Therefore we have that

$$
Q_{\mathrm{rad}}^{e}=-\dot{R}=-\frac{3}{2} k_{B} T_{e} n_{e}^{2}\left(A_{c p}^{\mathrm{eff}}+A_{c s}^{\mathrm{eff}}\right),
$$

where $Q_{\mathrm{rad}}^{e}=-\dot{R}$ is the value of the electron thermal energy lowering due to radiative recombination.

\section{Convective and heat flux losses}

After having considered an explicit form for each of the source terms, the electron energy equation (10) becomes

$$
\begin{aligned}
\lambda \frac{\partial^{2} T_{e}}{\partial z^{2}}= & \frac{3}{2} k_{B} u n_{e} \frac{\partial T_{e}}{\partial z}+\dot{R}-\sigma E^{2}+\frac{3}{2} n_{e} k_{B}\left(T_{e}\right. \\
& \left.-T_{g}\right) \frac{2 m_{e}}{M_{h}} \nu_{e f}-\frac{\partial \lambda}{\partial T_{e}}\left(\frac{\partial T_{e}}{\partial z}\right)^{2},
\end{aligned}
$$

since

$$
\boldsymbol{\nabla} \cdot \mathbf{q}_{e}=-\nabla\left(\lambda \nabla T_{e}\right)=-\frac{\partial \lambda}{\partial T_{e}}\left(\frac{\partial T_{e}}{\partial z}\right)^{2}-\lambda\left(\frac{\partial^{2} T_{e}}{\partial z^{2}}\right),
$$

where $\lambda$ is the electron thermal conductivity in argon. The values of $\sigma$ and $\lambda$ are taken from the calculations made by Devoto ${ }^{18,19}$ using the Chapman-Enskog theory. These transport coefficients show a good agreement with experimental measurements, ${ }^{20,21}$ so we have fitted them with analytical formulas.

The term $\left(\frac{3}{2}\right) k_{B} u n_{e} \partial T_{e} / \partial z$ in Eq. (14) represents an electron energy loss. This energy loss is due to the axial movement of the plasma (convective energy loss).

For symmetry reasons, we require $\left(\partial T_{e} / \partial z\right)_{z=0}=0$; then we have at $z=0$

$\left(\frac{\partial^{2} T_{e}}{\partial z^{2}}\right)_{z=0}=\left(\frac{3 n_{e}\left(\frac{m_{e}}{M_{h}}\right)\left(T_{e}-T_{g}\right) \nu_{e f}-\sigma E^{2}+\dot{R}}{\lambda}\right)_{z=0}>0$,

so there is a minimum in the electron temperature $T_{e}$ at the end $(z=0)$ of the plasma column.

The model equations are solved from the end of the plasma column. The exact position of the launcher from the end of the column $(z=0)$ depends on the operating conditions considered in the calculations. The definitive set of equations to solve has the form

$$
\begin{aligned}
\frac{\partial n_{p}}{\partial z}= & \frac{n_{e} n_{p}}{u} k_{s p}+\frac{n_{e}^{3}}{u} k_{c p}+\frac{n_{e}^{2}}{u} A_{c p}^{\text {eff }}-\frac{n_{e} n_{p}}{u}\left(k_{p c}+k_{p s}\right) \\
& -\frac{n_{p}}{u} A_{p s}^{\text {eff }} \\
\frac{\partial n_{e}}{\partial z}= & \frac{n_{e} n_{s}}{u} k_{s c}+\frac{n_{e} n_{p}}{u} k_{p c}-\frac{n_{e}^{3}}{u}\left(k_{c s}+k_{c p}\right) \\
& -\frac{n_{e}}{u}\left(A_{c s}^{\text {eff }}+A_{c p}^{\text {eff }}\right), \\
\frac{\partial^{2} T_{e}}{\partial z^{2}}= & \frac{3 k_{B} n_{e} u}{2 \lambda} \frac{\partial T_{e}}{\partial z}-\frac{\partial \lambda}{\partial T_{e}}\left(\frac{\partial T_{e}}{\partial z}\right)^{2} \frac{1}{\lambda}+\frac{\dot{R}}{\lambda}-\frac{\sigma E^{2}}{\lambda} \\
& +\frac{3}{2} n_{e} k_{B}\left(T_{e}-T_{g}\right) \frac{2 m_{e} \nu_{\mathrm{eh}}}{\lambda M_{h}} .
\end{aligned}
$$

As we already assumed that the total number of particles $n_{a}$ remains constant along the plasma column, we can eliminate the ground-level population density $n_{s}$ from Eqs. (17)-(19).

$$
n_{s}=n_{a}-n_{e}-n_{p} \text {. }
$$

When several particle species are present in the plasma, the total particle population density $n_{a}$ is fixed by the working pressure $\left(p_{g}=1 \mathrm{~atm}\right)$ through the Dalton law,

$$
p \cong\left(n_{e}+n_{a}\right) k_{B} T_{g} \cong n_{a} k_{B} T_{g} .
$$

Equations (17)-(19) can be conveniently adimensionalized making use of the new variables,

$$
\begin{aligned}
& y(1)=\frac{n_{p}(z)}{n_{p 1}}, \\
& y(2)=\frac{n_{e}(z)}{n_{e 1}}, \\
& y(3)=\frac{S(z)}{S_{1}}, \\
& y(4)=\frac{T_{e}(z)}{T_{e 1}},
\end{aligned}
$$

where $n_{p 1}, n_{e 1}, S_{1}$, and $T_{e 1}$ are the values of the different magnitudes at some point close to $z=0$, where $S(z)=\partial T_{e} / \partial z$ is the electron temperature gradient along the plasma column. Also, we use the new variable $Z=z / L$, where $L$ is the length of the plasma column. The initial conditions at the column end are: $n_{p}=10^{6} \mathrm{~cm}^{-3} ; n_{e}=3 \times 10^{13} \mathrm{~cm}^{-3} ; T_{e}=5000$ $\mathrm{K}$; and $S=0$. The system (17)-(19) has been solved using a Runge-Kutta-Verner method of fifth and sixth order with variable step.

\section{RESULTS AND DISCUSSION}

In this section the results from the hydrokinetic model are presented. The calculations were carried out at atmospheric pressure, a microwave frequency of $2450 \mathrm{MHz}$, and with a given amount, $P_{0}=P(0)$, of the total power delivered by the wave launcher to the plasma. The power value is related with the wave attenuation coefficient $\alpha$, electric conductivity of the plasma $\sigma$, and the effective value of the total 

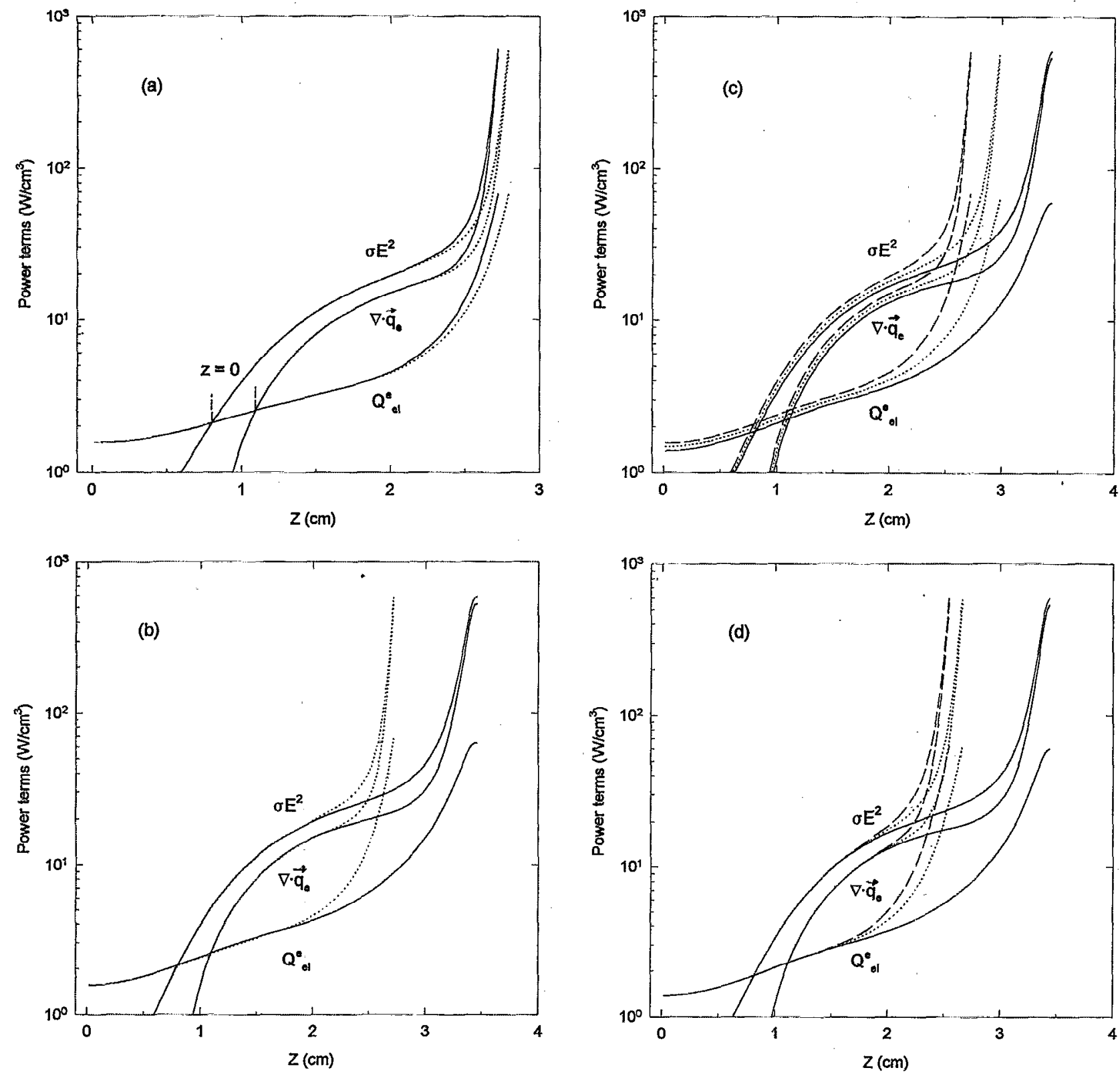

FIG. 4. Effects of different parameters on the axial power term profiles predicted by the hydrokinetic model. The dashed vertical lines in (a) show the real plasma column end for each parameter considered. This plasma column end must be taken into account in the rest of the figures. Each plot $\left(\sigma E^{2}, \nabla \cdot q_{e}\right.$, and $\left.Q_{\mathrm{el}}^{e}\right)$ contains different lines, each of them being related to the different parameters used: (a) gas flow $\phi:(一) 0.75$ and $(\cdots \cdots \cdots) 1.0 \ell /$ min; (b) inner tube radius $R:(-) 0.1$ and $(\cdots \cdots \cdots) 0.15 \mathrm{~cm}$; (c) neutral gas temperature $T_{g}:(\ldots \ldots) 1500,(\cdots \cdots \cdots) 1750$, and (-) $2000 \mathrm{~K}$; and (d) escape factor $\Lambda_{p s}:(-)$ $10^{-3},(\cdots \cdots \cdots) 10^{-4}$, and (---.) $10^{-6}$.

electric-field intensity within it. This relation may be expressed as the Joule losses: $P(0)=\left(\pi R^{2} / 2 \alpha\right)\left[\sigma E^{2}\left(n_{e}\right)\right]$. From the calculated $\sigma E^{2}$ values at gap level (see the following subsection) and the calculated $\alpha$ value (see Sec. II A), we are dealing with a total power of approximately $100 \mathrm{~W}$ at the launching gap.

The plasma forms near the gap and extends outward several $\mathrm{cm}$, depending upon input power and gas flow rate. For a given gas flow, the plasma length can be increased by increasing the HF power. ${ }^{16}$ In this article the power delivered by the wave launcher to the plasma is assumed constant. The working conditions (i.e., neutral gas temperature $T_{g}$, inner tube radius $R$, and inlet gas flow rate $\phi$ ) have been varied according to some commonly used experimental situations involving microwave discharges. Moreover, the radiation trapping effect $(p \rightarrow s)$ has been considered through different values of the escape factor $\Lambda_{p s}$.

The plasma and device characteristics assumed for carrying out the calculations were as follows:

(i) $\quad T_{g}$ (gas temperature) $=1500,1750$, and $2000 \mathrm{~K}$;

(ii) $\phi$ (gas flow rate) $=0.75$ and $1 \ell / \mathrm{min}$;

(iii) $R$ (inner tube radius) $=0.1$ and $0.15 \mathrm{~cm}$; and

(iv) $\Lambda_{p s}$ (escape factor for the resonance transition $p \rightarrow s)=10^{-3}, 10^{-4}$, and $10^{-5}$.

The assumption of assuming constant $T_{g}$ along the 

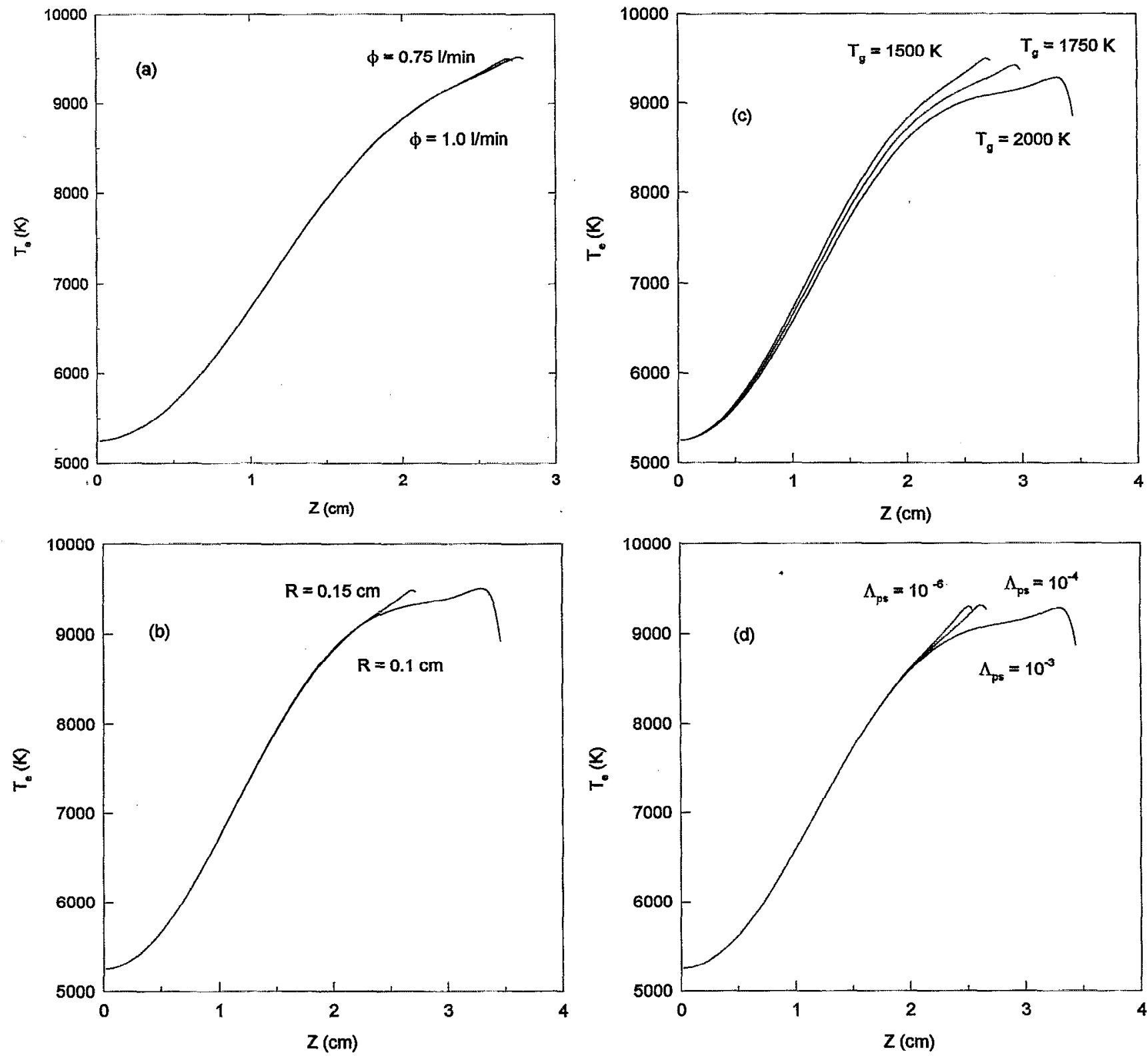

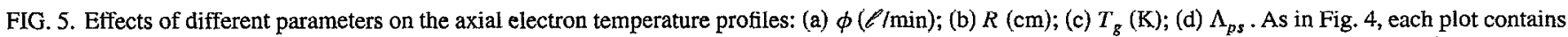
different lines, each of them being related to the different parameters considered.

plasma column is experimentally well corroborated, for this type of discharge, by recent measurements. 6,16

For clarity it should be remembered, when looking at the figures, that the power source is placed at the right-hand side of each plot.

\section{A. Power terms}

Figures 4(a)-4(d) show the most important gain and loss power density terms, i.e., ohmic heating, elastic losses, and the power loss related to the electron heat flux. We see that the power terms always decrease from the power source $(z \sim 2-3 \mathrm{~cm})$ to the plasma column end. Moreover, the real plasma end $(z \sim 1 \mathrm{~cm})$ is different from the column end considered in the calculations $(z=0)$ since at the end of the column (marked with a dashed vertical line), the ohmic power gain is under the elastic and heat flux losses. This has no real physical meaning and then the plasma cannot exist from this point down. The same is valid for the rest of the figures where these processes are presented for different plasma conditions. This remark must also be taken into account for all remaining figures. The effect of the inlet gas flow rate is evaluated in Fig. 4(a), which shows that the plasma column length becomes larger when the gas flow increases. This is due to the convective power losses, ( $\left.3 \phi k_{B} n_{e} / \pi R^{2}\right)\left(\partial T_{e} / \partial z\right)$; so when the flow rate $\phi$ increases, so do the convective losses and this has the effect of carrying the energy farther, so the plasma column extension increases a little.

Figure 4(b) shows the results of considering two different inner radii, $R=0.15$ and $0.1 \mathrm{~cm}$. Now, the higher $R$, the smaller the plasma length. This effect is again due to the 

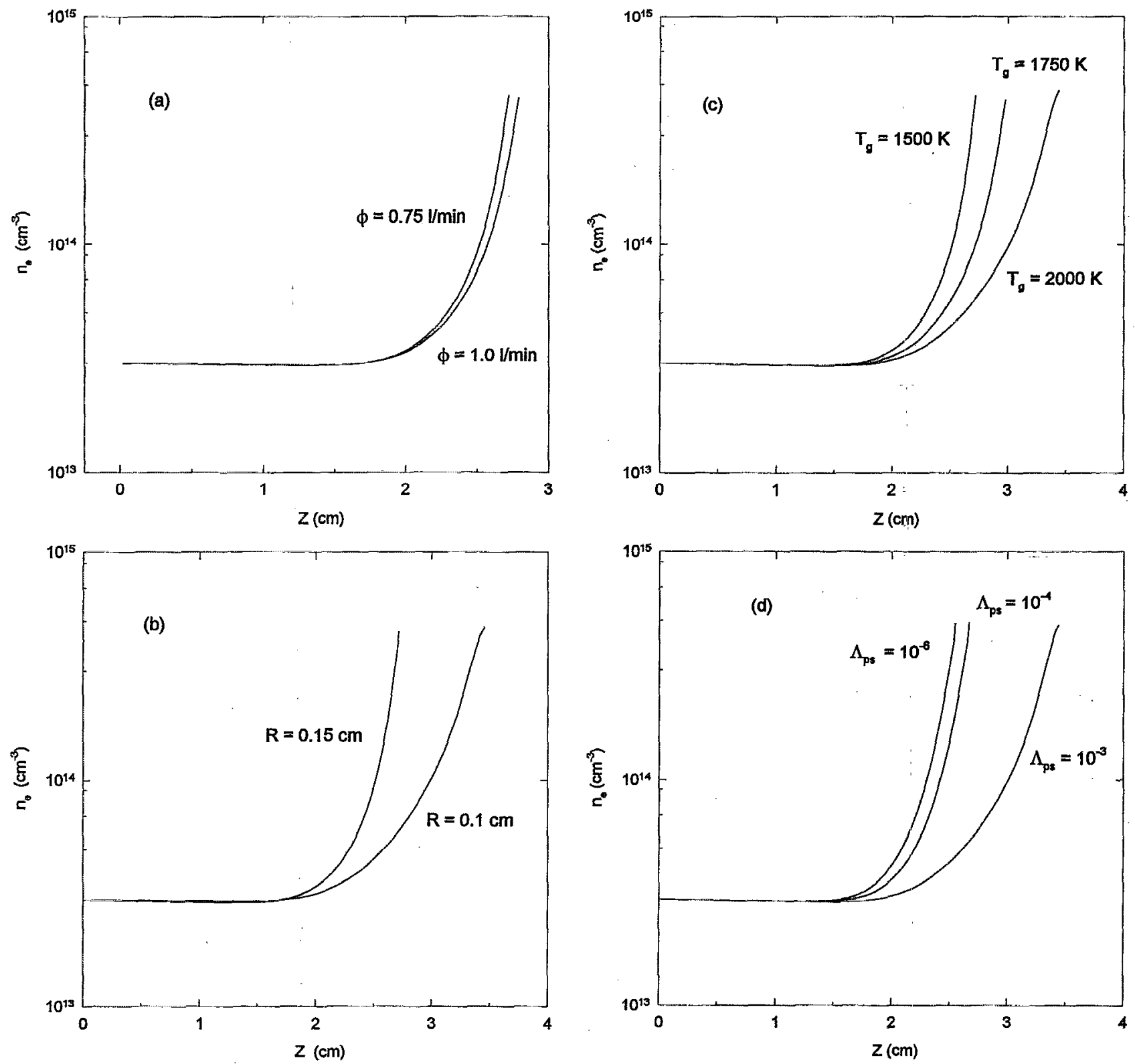

FIG. 6. Effects of different parameters on the axial electron density profiles: (a) $\phi(\ell / \mathrm{min})$; (b) $R$ (cm); (c) $T_{\&}$ (K); (d) $\Lambda_{p s}$. As in Fig. 4, each plot contains different lines, each of them being related to the different parameters considered.

convective losses, but here the effect is more important since the convective term has an inverse dependence on $R$.

Figure $4(\mathrm{c})$ shows the consequences of considering different gas temperatures $T_{g}$. The energy exchange terms associated with the elastic losses become more important when the temperature difference $\Delta T=T_{e}-T_{g}$, which is a measure of the departure from the thermodynamic equilibrium, increases. So at $T_{g}=1500 \mathrm{~K}$, the elastic losses are higher than at $T_{g}=2000 \mathrm{~K}$. When the gas temperature increases, the plasma length becomes longer.

The effect of the escape factor $\Lambda_{p s}$ is shown in Fig. 4(d). This is a particularly interesting feature of the model since $\Lambda_{p s}$ does not appear in the electron power balance equation [Eq. (7)] but it has an important indirect influence on the power terms and plasma length. If $\Lambda_{p s}$ is low $\left(10^{-4}, 10^{-6}\right)$, less radiation leaves the plasma and then the excited state $p$ is less depopulated. Another consequence of this is that the ionization mechanisms from level $p$ are more effective and then, for the same axial position, the electron density reaches a higher value than when $\Lambda_{p s}$ is $10^{-3}$. The consequence of this is that the higher the escape factor, the higher the plasma column length.

The effect of the four parameters considered can be summarized as follows: All the processes involving the increase of the power density loss, due to higher $n_{e}$ values, cause a decrement in the plasma column length.

Figures 4(a)-4(d) always show a plasma length of approximately $3-4 \mathrm{~cm}$. The plasma length found in this work agrees very well with the experimental length reported by different authors. ${ }^{4,16}$ Also, the rest of theoretical results obtained in this section are in good agreement with the experimental results reported in the references. ${ }^{2,16}$ 

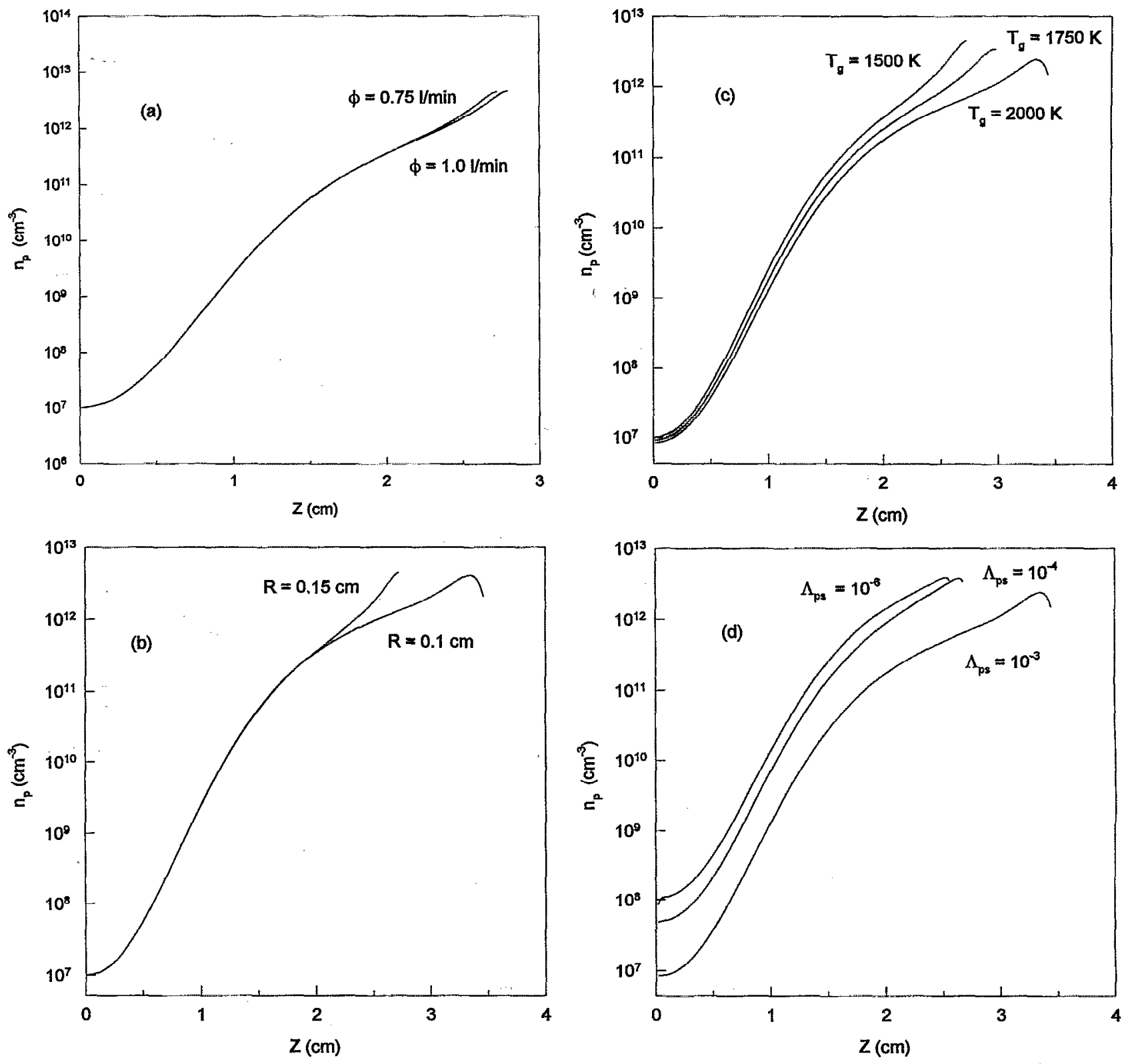

FIG. 7. Effects of different parameters on the axial profile of the first excited state: (a) $\phi(\ell / \mathrm{min})$; (b) $R$ (cm); (c) $T_{g}(\mathrm{~K})$; (d) $\Lambda_{p s}$. As in Fig. 4, each plot contain different lines, each of them being related to the different parameters considered.

It's also important to note that the electron heat flux is the primary energy loss process, followed by elastic collisions.

\section{B. Electron temperature}

The electron temperature behavior essentially follows the ohmic power profile for all the conditions considered. Figures 5(a)-5(d) show the axial electron temperature distribution for four different situations (see the figure captions). The hydrokinetic model shows then that close to the power source $(z \approx 3 \mathrm{~cm})$, the electron temperature reaches its highest value. Only the variation of the parameter $T_{g}$ has a remarkable influence on the axial behavior of $T_{e}$. The rest of parameters $\left(\phi, R\right.$, and $\left.\Lambda_{p s}\right)$ hardly change the value of the electron temperature close to the launcher.

\section{Electron and excited level densities}

The axial electron density distribution is shown in Figs. 6(a)-6(d) for four plasma conditions. Again, it is worth pointing out that the electron density equilibrium values (given by the Saha equation for the electron temperatures of the model) are two orders of magnitude higher than the electron density profiles obtained with the hydrokinetic model. Concerning the excited level density, its axial distribution is shown in Figs. 7(a)-7(d). The variation of the parameters $R$ and $\Lambda_{p s}$ produces a remarkable axial variation of $n_{p}$. As was expected, the effect on $n_{p}$ related with the variation of the escape factor $\Lambda_{p s}$ is the most important. The $p$ level density grows while getting closer to the power source, its equilibrium value (Boltzmann values) being just one order of magnitude higher. We see, in Fig. 7(d), that when plasma opacity 


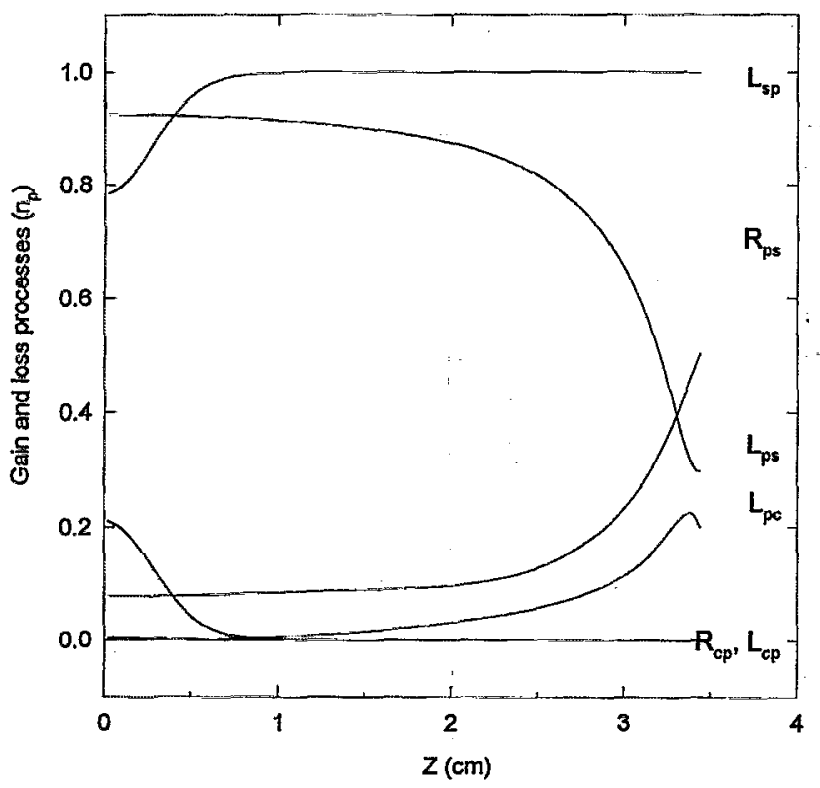

FIG. 8. Example of the relative importance of the various collisional and radiative transitions to the production of level $p$. The conditions of this plot are: $R=0.15 \mathrm{~cm} ; \phi=0.75 \mathscr{\ell} / \mathrm{min} ; T_{g}=2000 \mathrm{~K}$; and $\Lambda_{p s}=10^{-3}$.

increases (lower $\Lambda_{p s}$ ), the radiative deexcitation of level $p$ is not as effective. Therefore, at a given axial position, the smaller the escape factor, the greater the excited level density. In all plots, Figs. 7(a)-7(d) we see that the excited level population distribution follows a similar behavior to that of the ohmic heating. The calculation, at atmospheric pressure conditions, of the $p$ level population density, and the knowledge of the different kinetic processes that contribute to populating and depopulating it, is of interest for achieving new sources of metastable argon atoms. ${ }^{22}$ The theoretical values of $n_{e}$ obtained in our calculations are in reasonable agreement with available experimental measurements. ${ }^{5,16}$ On the other hand, there are no available experimental results, as far as we know, on the $4 s$ excited level of argon at atmospheric pressure. The $n_{p}$ values obtained show a sharp gradient along the plasma column.

In the rate equations (7) and (8) we have considered different collisional and radiative processes. The relative importance of the different volumetric transition rates $\left(L_{i j}, R_{i j}\right)$ for populating and depopulating the excited level $(p)$, and the continuum level $(c)$, are given in Figs. 8 and 9, respectively. The relative importance of the rate of population (or the rate of depopulation) of a level $(c, p$, or $s)$ was found by the ratio of this rate to the sum of all rates of population or depopulation of levels $c, p$, or $s$. This means that each transition for populating or depopulating a particular level $(c, p$, or $s$ ) will be characterized by two quantities: one that shows the relative importance of the transition to populating the upper level, and another that shows the relative importance of the transition to depopulating the lower level $\left(L_{p c}, L_{s c}, L_{s p}\right)$ or vice versa, i.e., to depopulating the upper level and to populating the lower level $\left(L_{c p}, L_{c s}, L_{p s}, R_{c p}, R_{c s}, R_{p s}\right)$. For example, the relative contribution of the quantity $L_{p c}$ to the loss of level $p$ is calculated by the ratio of this term to all terms that depopulate

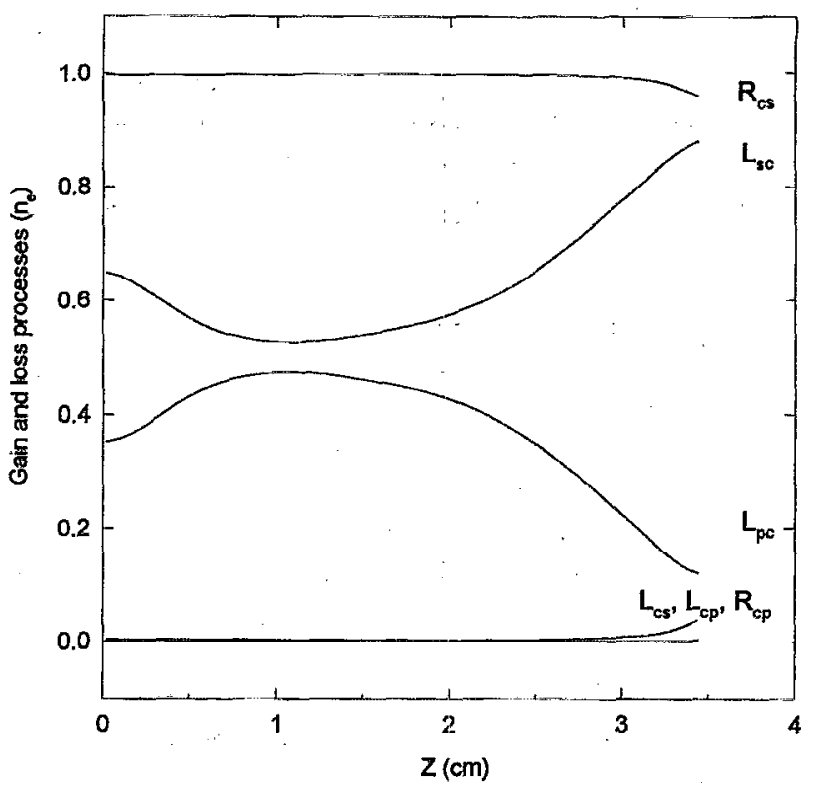

FIG. 9. The same calculation as in Fig. 8 but for the production of level $c$ (electrons).

level $p$. In the same way, the relative contribution of $L_{p c}$ to populating level $c$ is calculated by the ratio of this term to all terms that populate level $c$ In this way, the gain and loss terms are normalized separately to emphasize the kinetics associated with the different processes considered in the solution of the hydrokinetic model. In the same way, radiative processes are characterized by the symbol $R$. The subscripts in $L$ and $R$ have the same meaning as those associated with the volumetric transition rates (e.g., $L_{p c}=n_{e} n_{p} k_{p c}$ and $R_{c p}=n_{e}^{2} A_{c p}^{\text {eff }}$.

Figures 8 and 9 show the transition rate for populating and depopulating the excited level $(p)$, and the continuum level $(c)$, respectively. Figure 8 shows that the electronimpact excitation from level $s\left(n_{e} n_{s} k_{s p}\right)$ is the most important populating process of level $p$. Depending on what section of the plasma column is analyzed, the dominant volumetric rate of depopulation of level $p$ is the electronimpact deexcitation $\left(L_{p s}=n_{e} n_{p} k_{p s}\right)$ near the power source, or the spontaneous radiative deexcitation of level $p, R_{p s}$ $=n_{p} A_{p s}^{\text {eff }}$, as we go farther from the launcher. Figure 9 shows that the main volumetric rate of depopulation of the continuum $(c)$ is the two-body radiative recombination to the ground level $(s)\left(n_{e}^{2} A_{c s}^{\text {eff }}\right)$. Two different volumetric rates populate the continuum level: stepwise ionization from level $p$ and direct ionization from the ground level $(s)$. The direct ionization process $\left(L_{s c}=n_{e} n_{s} k_{s c}\right.$ ), clearly prevails in the plasma column second half, while along the first half $(1 \leqslant z \leqslant 1.5 \mathrm{~cm})$ both stepwise $\left(L_{p c}=n_{e} n_{p} k_{p c}\right)$ and direct ionization have very similar values.

The working conditions used in Figs. 8 and 9 are: a gas temperature of $2000 \mathrm{~K}$, inner tube radius of $0.15 \mathrm{~cm}$, gas flow rate of $0.75 \ell / \mathrm{min}$, and an optically thick plasma for the $p \rightarrow s$ radiative deexcitation with an escape parameter $\Lambda_{p s}=10^{-3}$.

In this simple manner we easily see the plasma kinetics for a particular set of conditions. 


\section{CONCLUSIONS}

In this article we have elaborated a hydrokinetic model that enables us to obtain the nonequilibrium axial behavior of the electron, first excited level $(p)$ densities, and electron temperature for a microwave-produced argon plasma at atmospheric pressure. The model shows, through the volumetric transition rate profiles, the relative importance of the different collisional and radiative processes in populating and depopulating levels $s, p$, and $c$. The three-level atomic structure is a simple approximation to a complicated atomic system. As discussed previously, there are several reasons to consider this approach.

The results obtained for argon show a reasonable good agreement with the available measurements. The electron density and temperature values obtained in our model show similar trends to those reported in the references in similar experimental situations. Moreover, the model predicts the axial behavior of the $p$ level ( $4 s$ state) population density. This density does not appear commonly in the experimental bibliography of MIP at atmospheric pressure conditions. The knowledge of this population density and its dependence on the absorbed power may be of some interest in understanding the physics of new sources of beams of metastable argon atoms in the thermal energy range. Also, they have interest regarding the microwave-induced argon plasma used in spectroanalytical analysis.

웅

\section{ACKNOWLEDGMENT}

Partial financial support of the DGICYT of the Spanish Ministry of Education and Science through Grant No. PB910847 and PB94-1457 is gratcfully acknowledged.
${ }^{\mathrm{t}}$ M. H. Abdallah, S. Coulombe, and J. M. Mermet, Spectrochim. Acta B 37, 583 (1982).

${ }^{2}$ M. Selby and G. M. Hieftje, Spectrochim. Acta B 42, 285 (1987).

${ }^{3}$ M. Moisan and Z. Zakrzewski, in Microwave Excited Plasmas, edited by M. Moisan and J. Pelletier (Elsevier, Amsterdam, 1992).

${ }^{4}$ M. Moisan, Z. Zakrzewski, and R. Pantel, J. Phys. D 12, 219 (1979); M. Moisan and Z. Zakrzewski, ibid. 24, 1025 (1991).

${ }^{5}$ M. Moisan, R. Pantel, and J. Hubert, Contrib. Plasma Phys. 30, 293 (1990).

${ }^{6}$ J. Cotrino, M. Sảez, M. C. Quintero, A. Menendez, E. Sánchez Uria, and A. Sanz-Medel, Spectrochim. Acta B 47, 425 (1992).

${ }^{7}$ Microwave Discharges. Fundamentals and Applications, edited by C. M. Ferreira and M. Moisan (Plenum, New York, 1993).

${ }^{8}$ H. Nowakowska, Z. Zakrzewski, and M. Moisan, J. Phys. D 22, 789 (1990).

${ }^{9}$ R. F. Whitmer and G. F. Herrmann, Phys. Fluids 9, 768 (1966).

${ }^{10} \mathrm{C}$. Boisse-Laporte, Microwave Discharges. Fundamentals and Applications, edited by C. M. Ferreira and M. Moisan (Plenum, New York, 1993).

${ }^{11}$ A. Granier, C. Boisse-Laporte, P. Leprince, J. Marec, and P. Nghiem, J. Phys. D 20, 204 (1987).

${ }^{12}$ W. L. Wiese, M. W. Smith, and B. M. Glennon, Atomic Transition Probabilities II: Sodium through Calcium, NSRDS-NBS 22 (National Bureau of Standards, Washington, DC, 1966).

${ }^{13}$ C. G. Braun and J. A. Kunc, Phys. Fluids 30, 499 (1987).

${ }^{14} \mathrm{~T}$. Hascgawa and H. Haraguchi, Spectrochim. Acta B 40, 1067 (1985).

${ }^{15} \mathrm{C}$. G. Braun and J. A. Kunc, Phys. Fluids 31, 671 (1988).

${ }^{16}$ A. Besner, M. Moisan, and J. Hubert, J. Anal. Atom. Spectrom. 3, 863 (1988).

${ }^{17}$ G. M. W. Kroesen, D. C. Schram, C. J. Timmermans, and J. C. M. de Haas, IEEE Trans. Plasma Sci. PS-18, 985 (1990).

${ }^{18}$ R. S. Devoto, Phys. Fluids 10, 354 (1967).

${ }^{19}$ R. S. Devoto, Phys. Fluids 16, 616 (1973).

${ }^{20}$ I. Bues, H. J. Patt, and J. Richter, Z. Angew. Phys. 22, 345 (1967).

${ }^{21}$ C. F. Knopp and A. B. Cambel, Phys. Fluids 9, 989 (1966).

${ }^{22}$ M. E. Bannister and J. L. Cecchi, J. Vac. Sci. Technol. A 12, 106 (1994). 